\title{
Cortisol and Cortisone Excretion from Infancy to Adult Life
}

\author{
By Merlyn C. Minick
}

Cortisol and cortisone excretion rates were determined simultaneously in 43 healthy infants, children and young adults. The results are compared to similar studies in 10 adults. A range of normal values is given. In terms of body weight, young people as compared with adults excrete significantly less free cortisol, but similar amounts of cortisone. (Metabolism 15: No. 4, April, 359-363, 1966)

T

HE PURPOSE of this communication is to record normal values for the excretion of free cortisol, cortisone and the ratio of cortisol to cortisone $(\mathrm{F} / \mathrm{E})$ excreted by infants, children and young adults. The data were obtained from 43 healthy subjects ( 22 males, 21 females) ranging in age from 3 months to 20 years. Ten additional subjects (all male) from 21 to 30 years of age were similarly studied for comparative purposes.

\section{SubJeCts AND METHODS}

Table 1 gives the age, weight and sex distribution of the young people studied. The table includes the group of adults against which comparisons were made. All subjects were eating normally and had not been exposed to known stressful circumstances prior to or during the collection of urine.

For the determination of cortisol and cortisone an adaptation of the method of Neher and Wettstein ${ }^{1}$ was employed. The method of urine collection from infants and data involving recovery of added steroids have been reported previously.2 The substances which were measured as cortisol and cortisone were further identified following acetylation by comparing their mobilities with known steroid acetates in various paper chromatographic systems.

4-C14 cortisol was applied to diapers containing urine and traces of fecal material. The diapers were then stored and processed as described.2 Since 4-C ${ }^{14}$ cortisone was not found, it was concluded that no significant conversion of cortisol to cortisone occurs during the collection of urine by the diaper method.

\section{Results}

Table 1 contains the data from which Figures 1,2 and 3 have been derived.

Figure 1 shows the excretion rates (mean) of cortisol and cortisone in the various age groups. A good correlation between urinary cortisol, cortisone and age exists between the ages of 3 months and 20 years, $(r=+0.785)$ and $(r=+0.760)$, respectively. From 21 through 30 years of age no significant correlation was found $(r=-0.342)$ and $(r=-0.294)$. All groups below

From the Department of Internal Medicine (Division of Endocrinology and Metabolism and the Metabolic Research Unit), The University of Michigan Medical School, Ann Arbor, Michigan.

Received for publication Dec. 30, 1965.

MerLyn C. Minick, M.S.: Research Associate, Division of Endocrinology and Metabolism and the Metabolism Research Unit, University Hospital, Ann Arbor, Mich. 


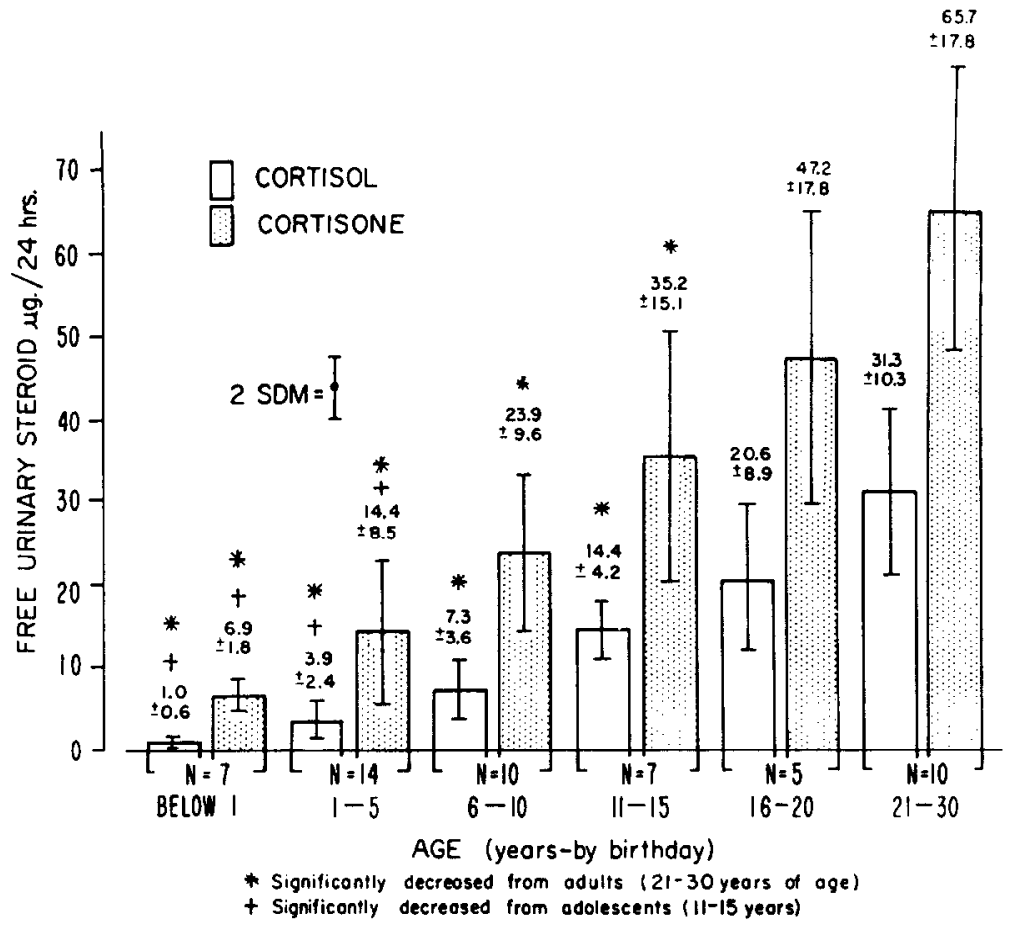

Fig. 1.

age 16 are significantly different from the adult group, the minimum group differences being highly significant $(\mathrm{P}<.005)$. All groups below age 6 are significantly different from the adolescents ( 11 to 15 years, $\mathrm{P}<.001$ ), while those from 6 to 10 years of age are not $(P>.05)$. The infants excrete significantly less cortisol than any other group $(\mathrm{P}<.01)$. This observation is in accord with that of Ulstrom et al. ${ }^{3}$ who found very low levels of cortisol in the urine of newborn infants.

Figure 2 represents the excretion rates expressed in $\mu \mathrm{g} . / \mathrm{Kg}$. body weight $/ 24$ hours. The correlation between cortisol and age is good $(r=+0.532)$. Cortisone excretion and age do not correlate $(\mathrm{r}=+0.022)$. The excretion of cortisol by infants, on a body weight basis, is also significantly less than any other group ( $\mathrm{min}$. diff. $\mathrm{P}<0.05$ ). With respect to cortisol excretion, none of the groups between the ages of 1 and 20 years are statistically different from each other. However, children below age 10 excrete significantly less cortisol than do adults (21-30 years). No significant differences exist between any of the groups with respect to cortisone excretion.

Significant differences in the excretory ratios at various ages are shown in Figure 3 ( $\mathrm{min}$. diff. $\mathrm{P}<.01$ ). The children below age 6 have excretory ratios which are different from those of age 11 and above (min. diff. P. $<001$ ) because they excrete relatively more cortisone than cortisol. 


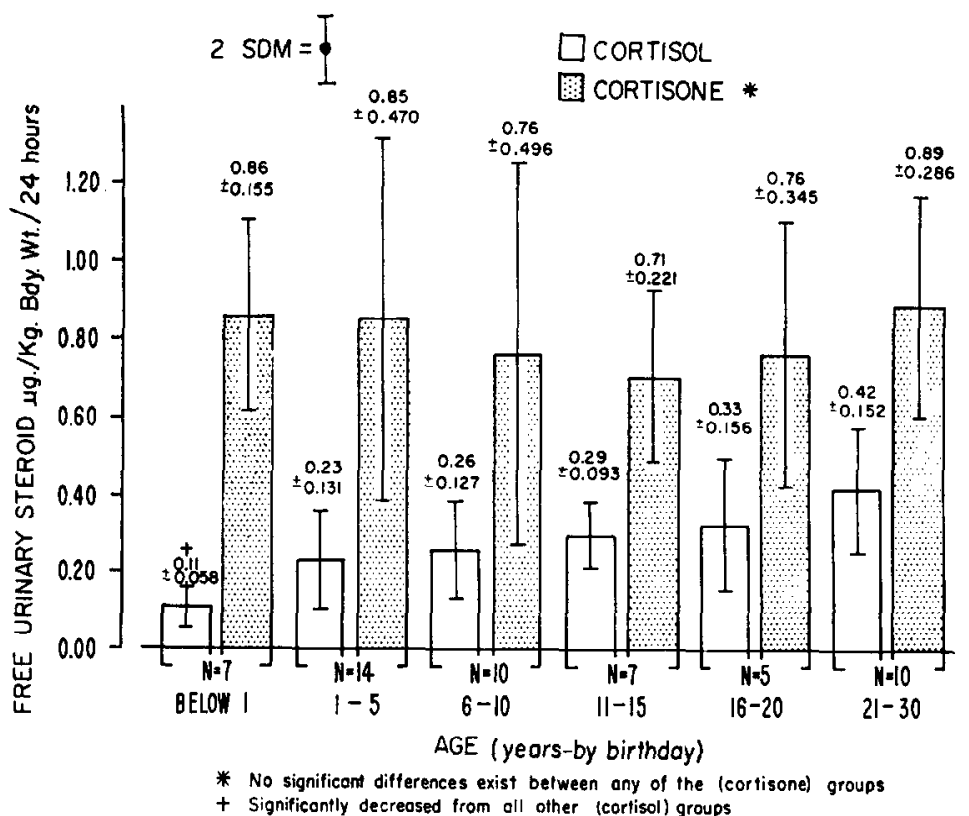

Fig. 2.

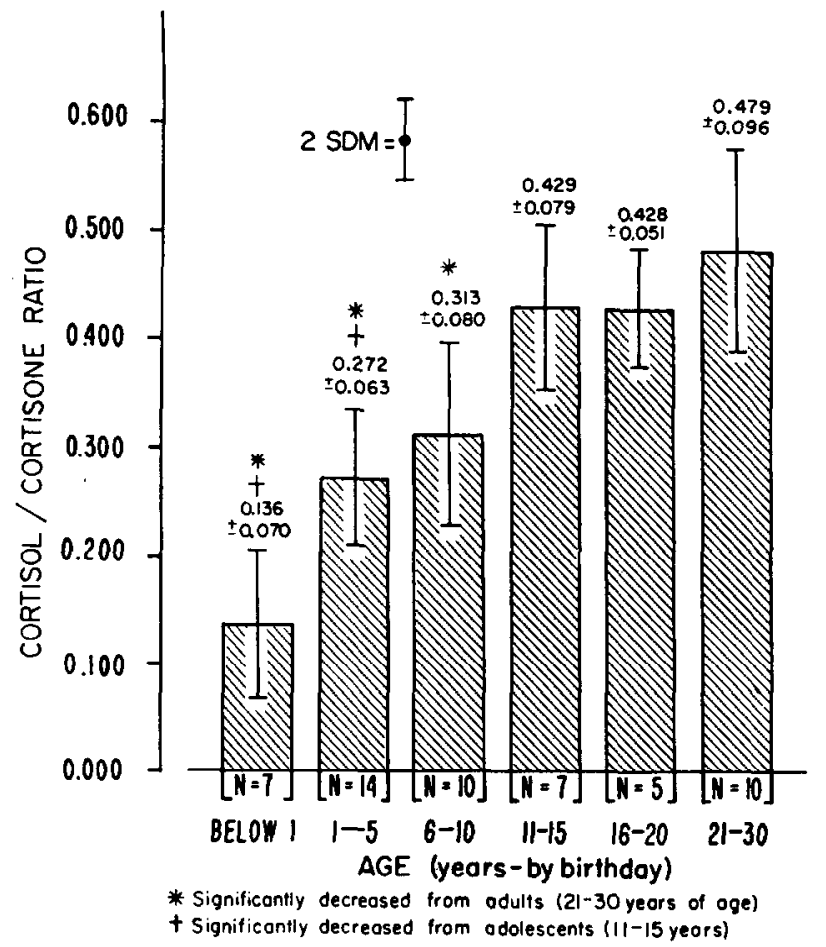

Fig. 3. 
Table 1.-Values for Cortisol and Cortisone Excretion on 53 Subjects

\begin{tabular}{|c|c|c|c|c|c|c|c|c|}
\hline No. & Suhjects & & $\begin{array}{l}\text { e } \\
\text { mo. }\end{array}$ & Sex & $\begin{array}{c}\text { Body } \\
\text { Weight } \\
\text { Ke. }\end{array}$ & $\begin{array}{c}\text { Cortisol } \\
\text { ug./24 } \\
\text { hrs. }\end{array}$ & $\begin{array}{c}\text { Cortisone } \\
\text { ug./24 } \\
\text { hrs. }\end{array}$ & $F / E$ \\
\hline 1. & C.H. ${ }^{*}$ & - & 3 & $\mathrm{M}$ & 5.1 & 0 & 5 & 0.000 \\
\hline 2. & C.S. & - & 4 & $\mathrm{~F}$ & 5.9 & 0.6 & 6 & 0.100 \\
\hline 3. & L.P. ${ }^{*}$ & - & 5 & $\mathrm{~F}$ & 7.2 & 0.5 & 5 & 0.100 \\
\hline 4. & P.J.P.* & - & 7 & $\mathbf{M}$ & 8.2 & 1 & 7 & 0.143 \\
\hline 5. & D.M. ${ }^{*}$ & - & 9 & $\mathrm{~F}$ & 10.0 & 2 & 7 & 0.286 \\
\hline 6. & B.B. & - & 11 & $\mathbf{M}$ & 10.9 & 1 & 8 & 0.125 \\
\hline 7. & B.E. & - & 11 & $F$ & 9.5 & 2 & 10 & 0.200 \\
\hline 8. & E.A. ${ }^{*}$ & 1 & - & $\mathrm{F}$ & 10.0 & 2 & 7 & 0.286 \\
\hline 9. & D.O.M. & 2 & 3 & $\mathbf{M}$ & 13.6 & 3 & 8 & 0.375 \\
\hline 10. & R.F.L. & 2 & 7 & M & 14.5 & 7 & 25 & 0.280 \\
\hline 11. & B.C. & 2 & 8 & $F$ & 11.8 & 5 & 16 & 0.313 \\
\hline 12. & K.E. & 2 & 9 & M & 13.6 & 1 & 7 & 0.143 \\
\hline 13. & K.S. & 2 & 10 & $\mathrm{~F}$ & 12.2 & 1 & 5 & 0.200 \\
\hline 14. & P.S. & 3 & 6 & $\mathrm{~F}$ & 15.0 & 4 & 16 & 0.250 \\
\hline 15. & K.M.H. & 4 & - & $\mathrm{M}$ & 20.5 & 1 & 4 & 0.250 \\
\hline 16. & P.C. & 4 & 6 & $\mathrm{M}$ & 16.8 & 4 & 15 & 0.267 \\
\hline 17. & C.D.M. & 4 & 9 & $\mathrm{~F}$ & 20.2 & 2 & 6 & 0.333 \\
\hline 18. & B.A.M. & 4 & 11 & $\vec{F}$ & 23.6 & 4 & 16 & 0.250 \\
\hline 19. & A.C.E. & 4 & 11 & $\mathrm{M}$ & 27.2 & 8 & 26 & 0.308 \\
\hline 20. & J.H. & 5 & - & M & 18.1 & 6 & 30 & 0.200 \\
\hline 21. & H.M. & 5 & 1 & M & 24.0 & 7 & 20 & 0.350 \\
\hline 22. & E.A.L. & 6 & 6 & $\mathrm{~F}$ & 22.7 & 4 & 9 & 0.444 \\
\hline 23. & E.J.L. & 6 & 7 & $\mathrm{~F}$ & 20.0 & 10 & 34 & 0.294 \\
\hline 24. & J.C.F. & 7 & 1 & M & 24.0 & 6 & 20 & 0.300 \\
\hline 25. & A.T. & 7 & 4 & F & 25.0 & 9 & 26 & 0.346 \\
\hline 26. & R.C.B. & 7 & 8 & $F$ & 25.4 & 5 & 20 & 0.250 \\
\hline 27. & K.T. & 8 & 6 & $F$ & 28.4 & 4 & 14 & 0.286 \\
\hline 28. & J.F. & 9 & - & M & 40.0 & 7 & 24 & 0.292 \\
\hline 29. & A.M.R. & 9 & 5 & $\mathrm{~F}$ & 36.3 & 6 & 18 & 0.333 \\
\hline 30. & D.E.B. & 10 & 4 & $\mathbf{M}$ & 31.8 & 6 & 36 & 0.167 \\
\hline 31. & M.A.B. & 10 & 10 & M & 36.5 & 16 & 38 & 0.421 \\
\hline 32. & M.J.B. & 11 & 10 & $F$ & 40.0 & 18 & 35 & 0.514 \\
\hline 33. & P.F. & 12 & - & $\mathbf{M}$ & 43.0 & 9 & 20 & 0.450 \\
\hline 34. & M.M.L. & 12 & 9 & $\mathrm{~F}$ & 44.2 & 12 & 32 & 0.375 \\
\hline 35. & R.H.H. & 13 & 7 & $\mathrm{~F}$ & 57.7 & 10 & 23 & 0.435 \\
\hline 36. & G.P.F. & 13 & 8 & $\mathbf{M}$ & 45.4 & 15 & 28 & 0.536 \\
\hline 37. & J.W.R. & 14 & 1 & M & 68.6 & 20 & 64 & 0.313 \\
\hline 38. & M.I.L. & 14 & 11 & $\mathrm{~F}$ & 48.1 & 17 & 45 & 0.378 \\
\hline 39. & J.L.R. & 16 & 5 & $F$ & 59.0 & 28 & 64 & 0.437 \\
\hline 40. & G.J.W. & 17 & - & $\mathbf{M}$ & 50.9 & 25 & 60 & 0.417 \\
\hline 41. & R.D.F. & 17 & 7 & $\mathbf{M}$ & 68.1 & 12 & 28 & 0.429 \\
\hline 42. & P.II. & 18 & - & $\mathbf{M}$ & 58.1 & 10 & 28 & 0.357 \\
\hline 43. & J.C. & 18 & - & M & 80.1 & 28 & 56 & 0.500 \\
\hline 44. & J.C.M. & 22 & - & $\mathbf{M}$ & 75.0 & 36 & 88 & 0.409 \\
\hline 45. & L.J.B. & 22 & - & $\mathbf{M}$ & 68.0 & 32 & 56 & 0.571 \\
\hline 46. & J.S.B. & 22 & - & $\mathbf{M}$ & 79.4 & 36 & 72 & 0.500 \\
\hline 47. & J.G.E. & 22 & - & $\mathbf{M}$ & 84.0 & 28 & 48 & 0.583 \\
\hline 48. & B.A.C. & 23 & & $\mathbf{M}$ & 84.1 & 20 & 44 & 0.454 \\
\hline 49. & J.A.S. & 25 & - & $\mathbf{M}$ & 68.1 & 24 & 80 & 0.300 \\
\hline 50. & J.J.P. & 25 & - & $\mathbf{M}$ & 78.6 & 45 & 85 & 0.529 \\
\hline 51. & J.S.S. & 26 & - & $\mathbf{M}$ & 62.1 & 28 & 64 & 0.437 \\
\hline 52. & D.E.M. & 27 & - & $\mathbf{M}$ & 67.7 & 48 & 80 & 0.600 \\
\hline 53. & M.C.M. & 30 & - & M & 89.0 & 16 & 40 & 0.400 \\
\hline
\end{tabular}

*Analyses from diapers (see text). 


\section{REFERENCES}

1. Neher, R., and Wettstein, A.: Physiochemical estimation of aldosterone in urine. J. Clin. Invest. 35:800-805, 1956.

2. Minick, M. C., and Conn, J. W.: Aldosterone excretion from infancy to adult life. Metabolism. 13:681-685, 1964.

3. Ulstrom, R. A., Colle, E., Burley, J. B., and Gunville, R.: Adrenocortical steroid metabolism in newborn infants. I. Urinary excretion of free and conjugated 17-hydroxy corticosteroids in normal full term infants. J. Clin. Endocrinol. \& Metab. 20:1066-1079, 1960. 